\title{
Corticothalamic Projections from the Rat Primary Somatosensory Cortex
}

\author{
Herbert P. Killackey and S. Murray Sherman \\ Department of Neurobiology and Behavior, University of California, Irvine, Irvine, California 92717, and Department of Neurobiology, State University of \\ New York, Stony Brook, New York 11794-5230
}

To study the cells of origin of corticothalamic inputs to the ventral posterior and posterior medial nuclei of the somatosensory thalamus in rats, we injected small aliquots of tracer into each nucleus and analyzed the pattern of retrograde labeling in the posteromedial barrel subfield of primary somatosensory cortex, which can be divided into barrel and nonbarrel zones. The ventral posterior nucleus is innervated by neurons in layer VIa of both zones, whereas the posterior medial nucleus is innervated by neurons in layers Vb and VIb of both zones with additional innervation from layer VIa of nonbarrel cortex. Thus, only the posterior medial nucleus receives a layer $\mathrm{Vb}$ input. Because the layer $\mathrm{Vb}$ input is interpreted as the initiation of a feedforward cortico-thalamo-cortical pathway, this implies that the target of the posterior medial nucleus, which includes the nonbarrel cortex, is a higher-order cortical area. We thus suggest that this cortical zone, which is classically considered part of the primary somatosensory cortex, should be reclassified as higher-order cortex.

Key words: thalamus; cortex; somatosensory; higher-order; first-order; barrel cortex; ventral posterior nucleus

\section{Introduction}

Within the posteromedial barrel subfield of primary somatosensory cortex in the rat (Woolsey and Van der Loos, 1970) is an unusual pattern of thalamocortical projections. This cortex includes the snout representation, in which each barrel represents a single mystacial vibrissa. For purposes of description, we divide this cortex into "barrel cortex" and "nonbarrel cortex." The former includes clusters of dense staining within layer IV, as revealed by various metabolic markers, whereas nonbarrel cortex is that into which the barrels are embedded, and this includes what has in the past been referred to as "septal" and "dysgranular" cortex (for review, see Killackey, 1983). Of interest in this context is the observation that the thalamic innervation of layer IV differs between barrel cortex and nonbarrel cortex; layer IV of the former is innervated by the main portion of the ventral posterior nucleus of the thalamus, whereas that of the latter is innervated by the posterior medial nucleus (Koralek et al., 1988; Lu and Lin, 1993). In addition, however, there is evidence that some innervation from the ventral posterior nucleus above and below layer IV spreads into nonbarrel cortex ( $\mathrm{Lu}$ and Lin, 1993). Furthermore, Pierret et al. (2000) distinguish a central "core" of the ventral posterior medial nucleus, which projects essentially only to barrel cortex, and a ventral "tail" region of the nucleus, which extends a projection into nonbarrel cortex and the second somatosensory area. Nonetheless, if we focus on the core region of the ventral posterior nucleus, it appears that its projection to layer IV is

Received April 22, 2003; revised June 5, 2003; accepted June 18, 2003.

This work was supported by National Science Foundation Grant BNS90-22168 and United States Public Health Service Grant EY03038. We thank Karen Good for help with collection of data.

Correspondence should be addressed to $S$. Murray Sherman, Department of Neurobiology, State University of New York, Stony Brook, NY 11794-5230. E-mail: s.sherman@sunysb.edu.

Copyright $\odot 2003$ Society for Neuroscience $\quad$ 0270-6474/03/237381-04\$15.00/0 effectively limited to barrel cortex, whereas that of the posterior medial nucleus innervates nonbarrel cortex, and thus there is a clear difference between the thalamocortical projections to layer IV between these thalamic nuclei.

The purpose of the present study is to clarify the extent to which the projections from the primary somatosensory cortex to these thalamic nuclei differ between barrel and nonbarrel regions. Although there have been previous studies of the corticothalamic projection from the primary somatosensory area in the rat (Chmielowska et al., 1989; Bourassa et al., 1995; Deschênes et al., 1998; Veinante et al., 2000), none to date has distinguished between the contributions of barrel versus nonbarrel cortex. To do so is the purpose of the present study.

\section{Materials and Methods}

Animals. Thirty adult Sprague Dawley rats of both sexes were used in this study. Rats were housed under the Association for Assessment and Accreditation of Laboratory Animal Care approved conditions. All experiments were conducted with previous approval of the University of California, Irvine, animal subjects committee and according to the guidelines established by the Society for Neuroscience.

Surgical procedures-tracer injections. Rats were anesthetized with ketamine hydrochloride $(100 \mathrm{mg} / \mathrm{kg})$ and xylazine $(3 \mathrm{mg} / \mathrm{kg})$ and placed in a stereotaxic apparatus. Aseptic conditions were maintained throughout the surgery. The animal's body temperature was kept at $37^{\circ} \mathrm{C}$ using a thermal pad. Respiration rates were monitored, and supplements of anesthesia were administered when needed.

Injections of either rhodamine- or fluorescein-conjugated microspheres (Lumafluor Corp., Naples, FL) were made from a vertical approach into the ventral posterior nucleus or the posterior medial nucleus using stereotaxic guidance. After a midline incision, $0.02-0.15 \mu \mathrm{l}$ of tracer was injected into each of the intended targets through a small hole in the skull. The rate and volume $(0.02-0.15 \mu \mathrm{l}$ per $300 \mathrm{sec})$ of injections were controlled by using calibrated micropipettes (tip diameter, 30-40 $\mu \mathrm{m})$ and a pressurized air injection system. Operated animals were 
placed on a thermal pad and observed until fully recovered and were then returned to home cage.

Histology. After $48 \mathrm{hr}$ of postinjection survival, animals were deeply anesthetized with Nembutol $(100 \mathrm{mg} / \mathrm{kg})$ and perfused transcardially with warm $\left(37^{\circ} \mathrm{C}\right) 0.1 \mathrm{M}$ Sorensen's PBS, $\mathrm{pH} 7.4$, followed by $2-4 \%$ paraformaldehyde, $0.1-0.5 \%$ glutaraldehyde, and $2 \%$ sucrose in phosphate buffer $(\mathrm{PB})$. Brains were removed from the skull, postfixed for 1-2 $\mathrm{hr}$, and cryoprotected in $30 \%$ sucrose in $\mathrm{PB}$ at $4^{\circ} \mathrm{C}$. Brains were sectioned on the freezing microtome at $40 \mu \mathrm{m}$ in the coronal plane. Brain sections were stored in $0.1 \mathrm{M} \mathrm{PB}$.

The tissue was processed for fluorescence microscopy as described previously (Katz et al., 1984), and a series of alternate coronal sections were reacted for $\mathrm{CO}$ according to the method described by Wong-Riley (1979). Sections were collected in and mounted from $0.1 \mathrm{M}$ phosphate buffer with $0.001 \%$ bisbenzamide as a counterstain to establish nuclear boundaries of the thalamus and reveal areal and cytoarchitectural features of the neocortex. Those sections reacted for $\mathrm{CO}$ were used to reveal the body surface map of the primary somatosensory cortex of the rat (Land and Simons, 1985) and to determine the boundary between the posterior medial (CO-poor) and ventral posterior (CO-rich) nuclei for analysis of injection sites.

Sections were mounted on chromalum-subbed slides, air-dried for 1-2 hr, defatted in xylene (30 sec), and coverslipped with flouromount (Atomergic Chemetals Corp., Farmingdale, NY). Sections reacted for CO were not dehydrated in alcohol to prevent differences in shrinkage between matching adjacent sections processed for fluorescence, which were also not dehydrated.

Analysis. Specimens were observed using a Leitz (Wetzlar, Germany) Orthoplan epifluorescent microscope equipped with the standard rhodamine (TRITC; BP, 55-560 nm; RKP, $580 \mathrm{~nm}$; LP, $580 \mathrm{~nm}$ ), fluorescein (FITC; BP, 450-490 nm; RKP, $510 \mathrm{~nm}$; LP, $525 \mathrm{~nm}$ ), and ultraviolet (UVA; BP 340-380 nm, RKP $400 \mathrm{~nm}$, LP $430 \mathrm{~nm}$ ) filter cubes. Photomicrographs were taken using a Leitz Vario-Orthomat 2 camera system. Bright-field photomicrographs were obtained using an auxiliary neutral density filter (ND2).

Sections through the mystacial vibrissae representation, or posteromedial barrel subfield, which were processed for CO histochemistry, were photographed through the microscope under bright-field illumination, and the adjacent sections were photographed at the same magnification under fluorescent illumination. Special care was taken to match pairs of photomicrographs so that landmarks in the tissue and magnification of the images matched precisely. Pairs of photomicrographs were confirmed to match by additional microscopic analysis of tissue borders and blood-vessel landmarks using dark-field illumination. With this method, the exact distribution of retrogradely labeled neurons can be determined with respect to relevant cytoarchitectonic features (barrels and septa) as revealed by corresponding (rich and poor) density of CO staining (Wong-Riley and Welt, 1980; Land and Simons, 1985).

\section{Results \\ Restriction of injection sites to the ventral posterior medial nuclei}

Only those cases in which individual injections were confined to either the central region of the ventral posterior nucleus or the posterior medial nucleus, and thus equivalent to the core region of Pierret et al. (2000), were used in the present analysis. Twelve cases with an injection into the ventral posterior nucleus and eight into the posterior medial nucleus were analyzed. Representative injection sites are illustrated in Figure 1, $A$ and $B$.

\section{Laminar origin of corticothalamic neurons}

Injection of microspheres into the ventral posterior nucleus results in retrogradely labeled neurons in primary somatosensory cortex evenly distributed in a single dense band confined to the top portion of layer VI (VIa). In what follows, we use the nomenclature established by Lorente de Nó (1922) in the mouse and Valverde et al. (1989) in the rat; that is, we subdivided layers V and VI into two parts, a top sublayer (a) and bottom sublayer (b). Occasionally, neurons in layers $\mathrm{VIb}$ and $\mathrm{Vb}$ are also labeled after an injection limited to the ventral posterior nucleus, but these are exceedingly rare and will not be discussed further. The distribution of neurons in layer VIa that are labeled from the ventral posterior nucleus is uninterrupted (Fig. 1C). The majority of labeled layer VIa neurons have the morphology of medium-sized pyramidal neurons with their presumed apical dendrites oriented vertically toward the cortical surface.

In contrast, injection of microspheres into the posterior medial nucleus results in a bilaminar distribution of labeled neurons. Figure 1, $D$ and $E$, shows two representative examples of labeling from the posterior medial nucleus. Figure $1 D$ shows a region through the somatosensory cortex showing regions of barrels separated by prominent nonbarrel cortex (barrel cortex and nonbarrel cortex are defined in the Introduction). Here, labeled neurons are evident in layers $\mathrm{Vb}$, VIa, and VIb. In layer VIa, however, the distribution of labeling is discontinuous, because it is limited to the nonbarrel cortex. This is unlike the distribution of continuous labeling from the ventral posterior nucleus in layer VIa (Fig. $1 C$ ). Figure $1 E$ shows barrel cortex in which any nonbarrel regions are too small to detect, and here there is a continuous and relatively dense band of labeled neurons in layer VIb and a less dense, yet continuous band of labeled neurons in layer $\mathrm{Vb}$. The morphology of the labeled neurons in layer $\mathrm{Vb}$ is clearly that of large pyramidal neurons with their apical dendrite oriented toward the cortical surface. The morphology of the smaller labeled neurons in layer VIb is considerably more variable. The presumed apical dendrite of many of these labeled neurons is oriented obliquely or horizontally.

\section{Discussion}

Figure 2 summarizes the pattern of corticothalamic projections that we have described that is also integrated with other related data. Previous studies have shown that thalamocortical afferents to layer IV from the ventral posterior nucleus are effectively limited to barrel cortex, whereas those from the posterior medial nucleus target only nonbarrel cortex (Koralek et al., 1988; Lu and Lin, 1993). Regarding corticothalamic innervation patterns, the ventral posterior nucleus receives afferents from neurons located in layer VIa that form a continuous band across both barrel and nonbarrel cortex. Little or no innervation of this thalamic nucleus arises from layers $\mathrm{Vb}$ or VIb. In contrast, the posterior medial nucleus receives afferents from neurons located in layers $\mathrm{Vb}$ and $\mathrm{VIb}$ that form a continuous band across both barrel and nonbarrel cortex. Additional projections to the posterior medial nucleus arise from layer VIa, but this is primarily limited to nonbarrel cortex. A key difference illustrated in Figure 2, and also reported previously (Veinante et al., 2000), is that the ventral posterior nucleus receives corticothalamic input only from layer VI, but the posterior medial nucleus receives such input from both layers $\mathrm{Vb}$ and VI.

\section{Comparison with visual pathways}

This pattern of corticothalamic innervation in the somatosensory system is remarkably similar to that described for the visual system. In rats, the lateral geniculate nucleus (which is analogous to the ventral posterior nucleus; see below) receives input from the primary visual cortex (which is analogous to the primary somatosensory cortex, especially the barrel cortex; see below) only from layer VI, whereas the lateral posterior-pulvinar complex (which is analogous to the posterior medial nucleus) receives such input from both layers V and VI (Bourassa and Deschênes, 1995). Fur- 

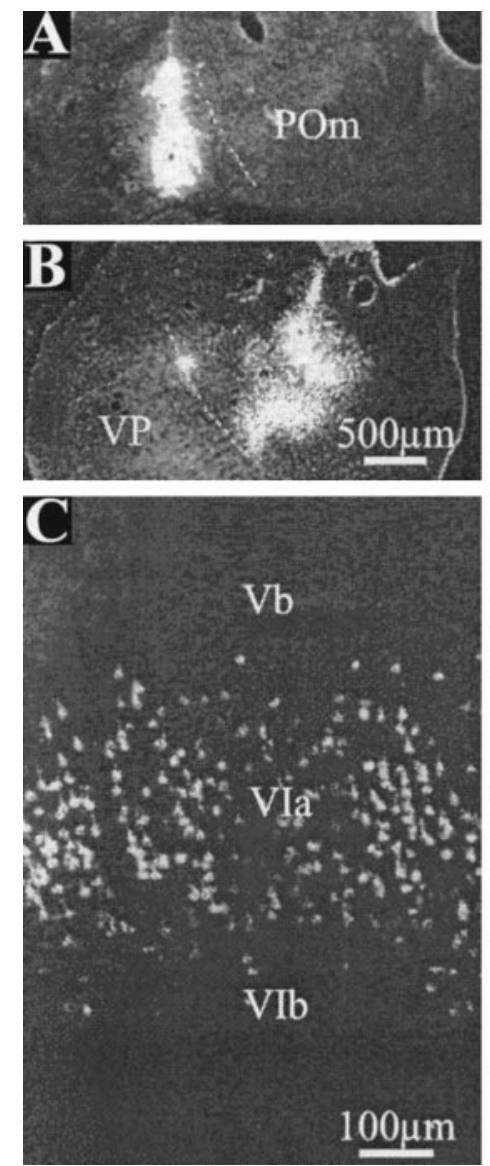
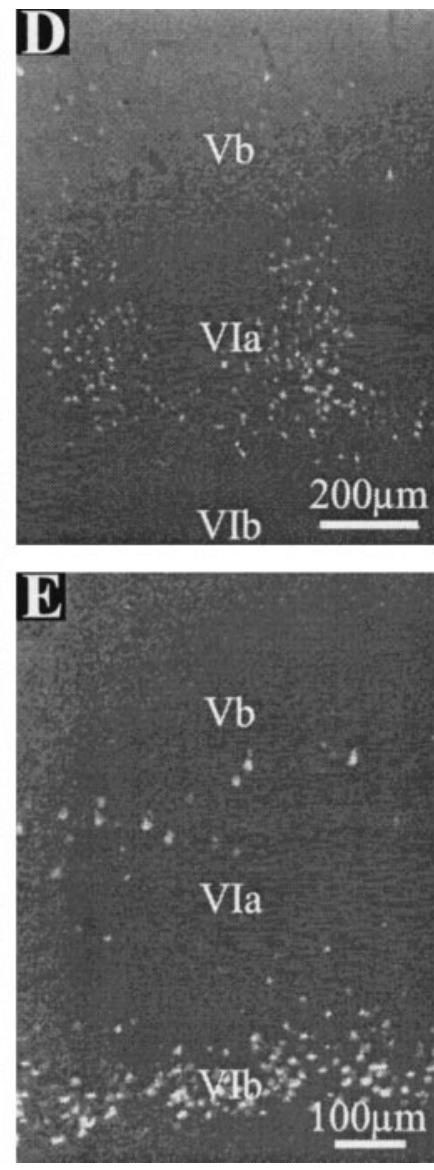

Figure 1. Injection sites and resultant retrograde labeling. $A, B$, Examples of injection sites limited to the ventral posterior or posterior medial nucleus. The dashed line indicates the border between these two nuclei. C, Retrograde labeling of primary somatosensory cortex after injection into the ventral posterior nucleus. The layers are indicated and were determined from staining with bisbenzamide and viewed through the appropriate filter (image not shown). Furthermore, alternate sections were stained with cytochrome oxidase to demonstrate the location of barrel and nonbarrel cortex as in $F$ and G.D, E, Retrograde labeling of primary somatosensory cortex after injection into the posterior medial nucleus. The layers are indicated and were determined as in $C$. $F$, $G$, Alternate sections showing cytochrome oxidase staining $(F)$ and retrograde labeling $(G)$. The arrows in $F$ indicate barrels and are repeated in $G$.

thermore, the layer VI input to the lateral geniculate nucleus arises mostly from the top part of the layer, whereas that to the lateral posterior-pulvinar complex arises from the lower part (Bourassa and Deschênes, 1995). Similarly, in cats, the lateral geniculate nucleus also receives primary visual cortical input only from layer VI, whereas the lateral posterior-pulvinar complex receives such input from layer V as well (Gilbert and Kelly, 1975; Abramson and Chalupa, 1985). Finally, in monkeys, the pattern is similar to that of rats and cats, because the lateral geniculate nucleus receives visual cortical input only from layer VI, and the lateral posterior-pulvinar complex is innervated as well from layer V (Lund et al., 1975; Fitzpatrick et al., 1994).

\section{Implications for functional organization of primary somatosensory cortex}

As noted above, a major difference between the ventral posterior and the posterior medial nuclei is that the latter receives input from layer $\mathrm{Vb}$ of cortex, although both receive input from layer VI. This is interesting in the context of the scheme proposed by Guillery and Sherman (Guillery, 1995; Sherman and Guillery, 2003). They suggested that thalamic relays can be divided into first and higher order. Both orders receive modulatory inputs from cortical layer VI. First-order relays transmit information from the periphery to cortex and thereby represent the first relay to cortex of such information. Examples are the lateral geniculate and ventral posterior nuclei, which transmit information from the optic tract and medial lemniscus, respectively. In contrast, higher-order relays serve as a transthalamic link between cortical areas (the route involving a projection from layer $\mathrm{Vb}$ of the first cortical area to a higher-order thalamic relay and then to a second cortical area). Examples of such higher-order relays are the lateral posterior nucleus and the pulvinar in the visual system and the posterior medial nucleus in the somatosensory system.

A logical implication of this hypothesis is that any cortical area in receipt of input from a higher-order thalamic relay is by definition a higher-order cortical area and not, for instance, a primary visual or somatosensory cortical area. This is particularly interesting in the context of Figure 2, because this requires that nonbarrel cortex, which is innervated by the posterior medial nucleus, is a higher-order cortical area, although it is conventionally regarded as part of the primary somatosensory cortex. We thus suggest that this conventional concept be reconsidered; namely, that what is regarded as primary somatosensory cortex in the rat is a mosaic of true primary cortex (i.e., barrel cortex) and a higher-order area (i.e., nonbarrel cortex).

It should be noted that previous authors have demonstrated other differences between barrel and nonbarrel cortex. For example, there are the differences in input from thalamus, because barrel cortex is innervated by the ventral posterior nucleus, whereas nonbarrel cortex is innervated by the posterior medial nucleus (Koralek et al., 1988; Lu and Lin, 1993). Also, there are differences in functional properties and intrinsic circuitry be- 
tween the two cortical zones (Chapin and Lin, 1984; Koralek et al., 1990; Kim and Ebner, 1999). However, to our knowledge, no one has yet suggested that this difference between areas includes a higherorder designation for nonbarrel cortex.

One final proviso needs to be considered. It is logical to think of the transthalamic corticocortical route involving layer $\mathrm{Vb}$ to be a feedforward pathway. This makes sense in regards to the layer Vb projection from barrel cortex to the posterior medial nucleus to nonbarrel cortex. However, what of the observation that nonbarrel cortex also sends a layer $\mathrm{Vb}$ projection to the posterior medial nucleus? This would be a feedback pathway if the relay cells innervated by this input projected back to nonbarrel cortex. However, as Figure 2 indicates, the posterior medial nucleus also projects to cortical areas beyond

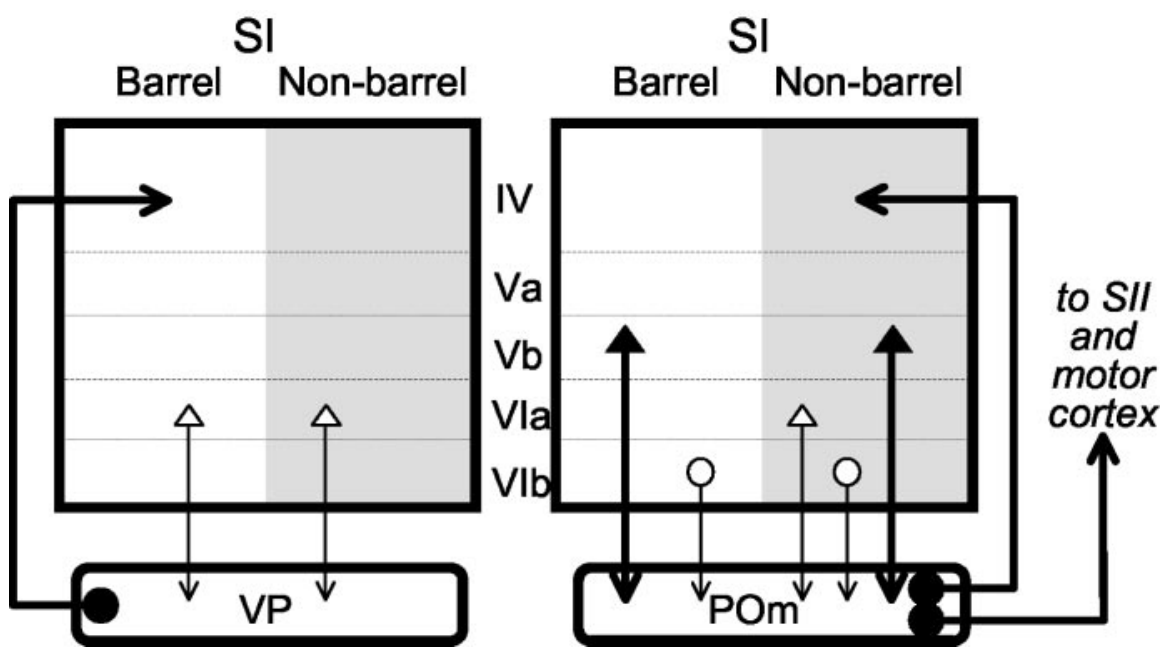

Figure 2. Schematic representation of relationships between thalamus and cortex involving the ventral posterior and posterior medial nuclei on the one hand and the primary somatosensory cortex on the other. See Results for details. SI, Primary somatosensory cortex; SII, secondary somatosensory cortex. the nonbarrel cortex, such as the second somatosensory cortex and motor cortex (Donoghue and Parham, 1983; Spreafico et al., 1987). It is thus possible that this input to the posterior medial nucleus from layer $\mathrm{Vb}$ of nonbarrel cortex also represents the initiation of a feedforward pathway, although this remains to be determined.

\section{References}

Abramson BP, Chalupa LM (1985) The laminar distribution of cortical connections with the tecto- and cortico-recipient zones in the cat's lateral posterior nucleus. Neuroscience 15:81-95.

Bourassa J, Deschênes M (1995) Corticothalamic projections from the primary visual cortex in rats: a single fiber study using biocytin as an anterograde tracer. Neuroscience 66:253-263.

Bourassa J, Pinault D, Deschênes M (1995) Corticothalamic projections from the cortical barrel field to the somatosensory thalamus in rats: a single-fibre study using biocytin as an anterograde tracer. Eur J Neurosci 7:19-30.

Chapin JK, Lin CS (1984) Mapping the body representation in the SI cortex of anesthetized and awake rats. J Comp Neurol 229:199-213.

Chmielowska J, Carvell GE, Simons DJ (1989) Spatial organization of thalamocortical and corticothalamic projection systems in the rat SmI barrel cortex. J Comp Neurol 285:325-338.

Deschênes M, Veinante P, Zhang ZW (1998) The organization of corticothalamic projections: reciprocity versus parity. Brain Res Brain Res Rev 28:286-308.

Donoghue JP, Parham C (1983) Afferent connections of the lateral agranular field of the rat motor cortex. J Comp Neurol 217:390-404.

Fitzpatrick D, Usrey WM, Schofield BR, Einstein G (1994) The sublaminar organization of corticogeniculate neurons in layer 6 of macaque striate cortex. Vis Neurosci 11:307-315.

Gilbert CD, Kelly JP (1975) The projections of cells in different layers of the cat's visual cortex. J Physiol (Lond) 163:81-106.

Guillery RW (1995) Anatomical evidence concerning the role of the thalamus in corticocortical communication: a brief review. J Anat 187:583-592.

Katz LC, Burkhalter A, Dreyer WJ (1984) Fluorescent latex microspheres as a retrograde neuronal marker for in vivo and in vitro studies of visual cortex. Nature 310:498-500.

Killackey HP (1983) The somatosensory cortex of the rodent. Trends Neurosci 6:425-429.

Kim U, Ebner FF (1999) Barrels and septa: separate circuits in rat barrels field cortex. J Comp Neurol 408:489-505.

Koralek KA, Jensen KF, Killackey HP (1988) Evidence for two complementary patterns of thalamic input to the rat somatosensory cortex. Brain Res 463:346-351.

Koralek KA, Olavarria J, Killackey HP (1990) Areal and laminar organization of corticocortical projections in the rat somatosensory cortex. J Comp Neurol 299:133-150.

Land PW, Simons DJ (1985) Cytochrome oxidase staining in the rat SmI barrel cortex. J Comp Neurol 238:225-235.

Lorente de Nó R (1922) La corteza cerebral del ratón. (Primera contribución. La corteza acústica.) Trabajos del Laboratorio de Investigaciones Biológicas de la Universidad de Madrid 20:41-78.

Lu SM, Lin RC (1993) Thalamic afferents of the rat barrel cortex: a lightand electron-microscopic study using Phaseolus vulgaris leucoagglutinin as an anterograde tracer. Somatosens Mot Res 10:1-16.

Lund JS, Lund RD, Hendrickson AE, Bunt AH, Fuchs AF (1975) The origin of efferent pathways from the primary visual cortex, area 17 , of the macaque monkey as shown by retrograde transport of horseradish peroxidase. J Comp Neurol 164:287-303.

Pierret T, Lavallée P, Deschênes M (2000) Parallel streams for the relay of vibrissal information through thalamic barreloids. J Neurosci 20:7455-7462.

Sherman SM, Guillery RW (2003) The role of thalamus in the flow of information to cortex. Philos Trans R Soc Lond B Biol Sci 357:1695-1708.

Spreafico R, Barbaresi P, Weinberg RJ, Rustioni A (1987) SII-projecting neurons in the rat thalamus: a single- and double-retrograde-tracing study. Somatosens Res 4:359-375.

Valverde F, Facal-Valverde MV, Santacana M, Heredia M (1989) Development and differentiation of early generated cells of sublayer VIb in the somatosensory cortex of the rat: a correlated Golgi and autoradiographic study. J Comp Neurol 290:118-140.

Veinante P, Lavallée P, Deschênes M (2000) Corticothalamic projections from layer 5 of the vibrissal barrel cortex in the rat. J Comp Neurol 424:197-204.

Wong-Riley M (1979) Changes in the visual system of monocularly sutured or enucleated cats demonstrable with cytochrome oxidase histochemistry. Brain Res 171:11-28.

Wong-Riley MT, Welt C (1980) Histochemical changes in cytochrome oxidase of cortical barrels after vibrissal removal in neonatal and adult mice. Proc Natl Acad Sci USA 77:2333-2337.

Woolsey TA, Van der Loos H (1970) The structural organization of layer IV in the somatosensory region (SI) of mouse cerebral cortex. The description of a cortical field composed of discrete cytoarchitectonic units. Brain Res 17:205-242. 